\title{
Protein expression of prenyltransferase subunits in postmortem schizophrenia dorsolateral prefrontal cortex
}

\author{
Anita L. Pinner', Toni M. Mueller', Khaled Alganem², Robert McCullumsmith ${ }^{2}$ and James H. Meador-Woodruff ${ }^{1}$
}

\begin{abstract}
The pathophysiology of schizophrenia includes altered neurotransmission, dysregulated intracellular signaling pathway activity, and abnormal dendritic morphology that contribute to deficits of synaptic plasticity in the disorder. These processes all require dynamic protein-protein interactions at cell membranes. Lipid modifications target proteins to membranes by increasing substrate hydrophobicity by the addition of a fatty acid or isoprenyl moiety, and recent evidence suggests that dysregulated posttranslational lipid modifications may play a role in multiple neuropsychiatric disorders, including schizophrenia. Consistent with these emerging findings, we have recently reported decreased protein S-palmitoylation in schizophrenia. Protein prenylation is a lipid modification that occurs upstream of S-palmitoylation on many protein substrates, facilitating membrane localization and activity of key intracellular signaling proteins. Accordingly, we hypothesized that, in addition to palmitoylation, protein prenylation may be abnormal in schizophrenia. To test this, we assayed protein expression of the five prenyltransferase subunits (FNTA, FNTB, PGGT1B, RABGGTA, and RABGGTB) in postmortem dorsolateral prefrontal cortex from patients with schizophrenia and paired comparison subjects ( $n=13$ pairs). We found decreased levels of FNTA (14\%), PGGT1B (13\%), and RABGGTB (8\%) in schizophrenia. To determine whether upstream or downstream factors may be driving these changes, we also assayed protein expression of the isoprenoid synthases FDPS and GGPS1 and prenylation-dependent processing enzymes RCE and ICMT. We found these upstream and downstream enzymes to have normal protein expression. To rule out effects from chronic antipsychotic treatment, we assayed FNTA, PGGT1B, and RABGGTB in the cortex from rats treated long-term with haloperidol decanoate and found no change in the expression of these proteins. Given the role prenylation plays in localization of key signaling proteins found at the synapse, these data offer a potential mechanism underlying abnormal protein-protein interactions and protein localization in schizophrenia.
\end{abstract}

\section{Introduction}

Altered neurotransmission is central to the pathophysiology of schizophrenia. Normal neurotransmission depends on regulation of receptor membrane localization and protein-protein interactions that regulate intracellular signaling activity ${ }^{1,2}$. Posttranslational modifications (PTMs), including lipid modification of proteins, have been shown to

\footnotetext{
Correspondence: Anita L. Pinner (anitapinner@uabmc.edu)

'Department of Psychiatry and Behavioral Neurobiology, University of Alabama at Birmingham, Birmingham, AL 35294-0021, USA

${ }^{2}$ Department of Neurosciences, University of Toledo, Toledo, $\mathrm{OH} 43614-2598$, USA
}

regulate neuronal functions and intracellular pathways by facilitating dynamic protein-protein interactions at membranes $^{3-5}$. Altered posttranslational lipid modifications may mechanistically contribute to intracellular signaling abnormalities reported in schizophrenia ${ }^{6}$.

Posttranslational lipid modifications include the enzymatic addition of an isoprenyl group such as farnesyl or geranylgeranyl (collectively called prenylation), or a fatty acid moiety, such as a palmitoyl or myristoyl group. Dysregulated lipid modifications of proteins have been implicated in neuropsychiatric disorders, including Alzheimer's disease ${ }^{7}$, Huntington's disease ${ }^{8,9}$, and in a mouse 
model of schizophrenia ${ }^{10}$. Abnormal lipid modifications have been reported in schizophrenia in dorsolateral prefrontal cortex (DLPFC), including decreased protein Spalmitoylation $^{11}$ and altered levels of a key $\mathrm{N}$ myristoylated protein ${ }^{12}$. While S-palmitoylation, N-myristoylation, and prenylation pathways can act independently, in some cases combinations of these lipid modifications are necessary for efficient membrane targeting, protein-protein interactions, and conformational dynamics of essential intracellular signaling proteins, such as heterotrimeric G-proteins and small monomeric GTPases $^{4,5,13-16}$. Abnormal G-protein signaling has been implicated in schizophrenia ${ }^{6,17-19}$, and heterotrimeric Gprotein subunits have been shown to require modifications by each one of these lipid modifications ${ }^{20-24}$. Dysregulated prenylation could be a mechanism contributing to this illness via altered G-protein signaling.

Prenylation involves the addition of either farnesyl or geranylgeranyl isoprenoid group(s). Three prenylation enzymes are responsible for the thioether linkage of isoprenoid moieties-15-carbon farnesyl pyrophosphate (FPP) or 20-carbon geranylgeranyl pyrophosphate (GGPP) - to C-terminal cysteines: farnesyl transferase (FTase), geranylgeranyl transferase I (GGTase I), and geranylgeranyl transferase II (GGTase II) ${ }^{25,26}$. Each enzyme is comprised of an $\alpha$ and a $\beta$ subunit. FTase and GGTase I have the same $\alpha$ subunit, FNTA, but have different $\beta$ subunits: FNTB is the FTase $\beta$ subunit, and PGGT1B the $\beta$ subunit of GGTase I. GGTase II, which specifically geranylgeranylates Rab family proteins, is made of the RABGGTA and RABGGTB subunits ${ }^{26}$. The addition of isoprenyl moieties leads to increased protein hydrophobicity, which facilitates targeted localization to membranes, lateral movement within membranes, and substrate conformational changes that can influence dynamic protein-protein interactions ${ }^{15,16}$.

Given that prenylation occurs upstream of Spalmitoylation $^{27}$, which is decreased in schizophrenia ${ }^{11}$, and facilitates the membrane targeting and resulting protein-protein interactions of key molecules associated with dynamic intracellular signaling ${ }^{4,5,28}$, we hypothesized that farnesylation and/or geranylgeranylation is also dysregulated in schizophrenia. Our own bioinformatic analysis of publically available datasets reflects a pattern of transcript expression differences for prenylationassociated enzymes and substrates in schizophrenia that further suggests dysregulation of this pathway. Accordingly, we assayed protein expression of the prenyltransferases subunits FNTA, FNTB, PGGT1B, RABGGTA, and RABGGTB in DLPFC from schizophrenia and matched comparison subjects. To further characterize the regulation of this modification, we also assayed protein expression of the upstream isoprenoid synthases farnesyl diphosphate synthase (FDPS) and geranylgeranyl pyrophosphate synthase (GGPS1) and the prenylation-dependent downstream processing enzymes Ras-converting enzyme (RCE) and isoprenylcysteine carboxyl methyltransferase (ICMT) in the same subjects. To identify the potential effects of antipsychotic treatment, we measured enzymes that were found altered in schizophrenia in the cortex from rats chronically treated with haloperidol decanoate.

\section{Methods and materials}

\section{Human subjects}

Samples of DLPFC (Brodmann area (BA) 9/46) from schizophrenia and matched comparison subjects $(n=13$ pairs) were obtained from the from the Mount Sinai School of Medicine (MSSM) NIH Brain and Tissue Repository (Table 1) as previously described ${ }^{12,29}$. Neuropathological examination of all subjects was conducted, and each subject's medical history was reviewed extensively; detailed information regarding assessment is available at http:// icahn.mssm.edu/research/labs/neuropathology-and-brainbanking/neuropathology-evaluation. Subjects with previous drug or alcohol abuse, coma $>6 \mathrm{~h}$, suicide, or any evidence of neurodegenerative disease were excluded from the study. Next of kin consent was obtained for each subject. Subjects with schizophrenia all met Diagnostic and Statistical Manual of Mental Disorders, Third Edition-Revised criteria, diagnosed by at least two clinicians, with documented onset of psychosis prior to 40 years of age, and a minimum of 10 years hospitalization for the illness. Comparison subjects were similarly evaluated and free of any neurological or psychiatric conditions. Based on our previous protein postmortem studies, power analysis determined that this sample size was adequate to detect a moderate effect size $\geq 0.3(\alpha=0.05, \beta=0.2)$. We performed data analyses assuming equal variance as the subjects were matched pairs. Experimenters were blinded until data analyses.

\section{Antipsychotic-treated rats}

Animal studies and procedures were performed in accordance to institutional guidelines and approved by the Institutional Animal Care and Use Committee of the University of Alabama at Birmingham. Twenty male Sprague-Dawley rats $(250 \mathrm{~g})$ were housed in pairs during the 9-month course of the study. Haloperidol deconoate $(28.5 \mathrm{mg} / \mathrm{kg}, n=10)$ or vehicle (sesame oil, $n=10)$ was administered every 3 weeks via intramuscular injection for a total of 12 injections ${ }^{30,31}$. Animals were sacrificed by rapid decapitation, and the brains were harvested immediately. The right frontal cortex was dissected on wet ice, snap frozen in liquid nitrogen, and stored at $-80^{\circ} \mathrm{C}$. Sample sizes were determined by previous studies, experimenters were blinded until data analyses, and sample groups were randomized. 
Table 1 Paired subject demographics.

\begin{tabular}{|c|c|c|c|c|}
\hline Pair & Subject & Sex/age & $\mathrm{pH}$ & PMI, h \\
\hline \multirow[t]{2}{*}{1} & Comparison & M/95 & 6.53 & 4.1 \\
\hline & Schizophrenia & M/97 & 6.50 & 9.3 \\
\hline \multirow[t]{2}{*}{2} & Comparison & $F / 66$ & 6.85 & 22.6 \\
\hline & Schizophrenia & $F / 62$ & 6.74 & 23.7 \\
\hline \multirow[t]{2}{*}{3} & Comparison & $\mathrm{F} / 73$ & 6.98 & 3.0 \\
\hline & Schizophrenia & $\mathrm{F} / 70$ & 6.51 & 13.2 \\
\hline \multirow[t]{2}{*}{4} & Comparison & $\mathrm{M} / 70$ & 6.10 & 6.7 \\
\hline & Schizophrenia & $\mathrm{M} / 70$ & 6.35 & 7.2 \\
\hline \multirow[t]{2}{*}{5} & Comparison & $\mathrm{F} / 74$ & 6.32 & 4.8 \\
\hline & Schizophrenia & $\mathrm{F} / 75$ & 6.49 & 21.5 \\
\hline \multirow[t]{2}{*}{6} & Comparison & $M / 73$ & 6.17 & 14.9 \\
\hline & Schizophrenia & $M / 73$ & 6.50 & 7.9 \\
\hline \multirow[t]{2}{*}{7} & Comparison & $\mathrm{F} / 80$ & 6.63 & 3.8 \\
\hline & Schizophrenia & $F / 81$ & 6.67 & 15.1 \\
\hline \multirow[t]{2}{*}{8} & Comparison & $\mathrm{F} / 79$ & 6.38 & 5.0 \\
\hline & Schizophrenia & $\mathrm{F} / 77$ & 6.01 & 26.1 \\
\hline \multirow[t]{2}{*}{9} & Comparison & $M / 76$ & 6.32 & 10.1 \\
\hline & Schizophrenia & $\mathrm{M} / 80$ & 6.37 & 9.7 \\
\hline \multirow[t]{2}{*}{10} & Comparison & $F / 85$ & 7.27 & 2.9 \\
\hline & Schizophrenia & $F / 84$ & 6.80 & 15.4 \\
\hline \multirow[t]{2}{*}{11} & Comparison & M/93 & 6.28 & 8.0 \\
\hline & Schizophrenia & M/92 & 6.67 & 21.9 \\
\hline \multirow[t]{2}{*}{12} & Comparison & $F / 89$ & 6.72 & 4.2 \\
\hline & Schizophrenia & $F / 89$ & 6.20 & 17.7 \\
\hline \multirow[t]{4}{*}{13} & Comparison & $\mathrm{M} / 75$ & 6.43 & 2.3 \\
\hline & Schizophrenia & $M / 78$ & 6.64 & 9.6 \\
\hline & Comparison $(n=13)$ & $79.1 \pm 8.9$ & $6.5 \pm 0.3$ & $7.1 \pm 5.8$ \\
\hline & Schizophrenia $(n=13)$ & $79.1 \pm 9.7$ & $6.5 \pm 0.2$ & $15.3 \pm 6.5$ \\
\hline
\end{tabular}

$P M I$ postmortem interval, $F$ female, $M$ male

\section{Tissue homogenization}

Tissue samples from both human subjects and rats were homogenized in ice-cold homogenization buffer $(5 \mathrm{mM}$ Tris- $\mathrm{HCl} \mathrm{pH} 7.5,0.32 \mathrm{M}$ sucrose) supplemented with protease and phosphatase inhibitor tablets (Complete Mini, EDTA-free and PhosSTOP; Roche Diagnostics, Mannheim, Germany), using A Power Gen 125 (ThermoFisher Scientific, Rockford, Illinois) homogenizer at speed setting 5 for $60 \mathrm{~s}$. A BCA Protein Assay Kit (ThermoFisher Scientific) was used to determine protein concentration, and samples were stored at $-80^{\circ} \mathrm{C}$.
Table 2 Antibodies used for western blot analyses.

\begin{tabular}{llllll}
\hline $\begin{array}{l}\text { Target } \\
\text { protein }\end{array}$ & $\begin{array}{l}\text { Host } \\
\text { species }\end{array}$ & Dilution & Incubation & Company & Catalog \# \\
\hline FNTA & Rabbit & $1: 1000$ & $16 \mathrm{~h}, 4^{\circ} \mathrm{C}$ & Abcam & ab109738 \\
FNTB & Rabbit & $1: 5000$ & $16 \mathrm{~h}, 4^{\circ} \mathrm{C}$ & Abcam & ab109625 \\
RABGGTA & Rabbit & $1: 500$ & $16 \mathrm{~h}, 4^{\circ} \mathrm{C}$ & Abcam & ab118781 \\
RABGGTB & Mouse & $1: 1000$ & $16 \mathrm{~h}, 4^{\circ} \mathrm{C}$ & Abnova & H00005876- \\
& & & & & M02 \\
PGGT1B & Mouse & $1: 1000$ & $16 \mathrm{~h}, 4^{\circ} \mathrm{C}$ & Abnova & H00005229- \\
& & & & & M02 \\
FDPS & Rabbit & $1: 1000$ & $16 \mathrm{~h}, 4^{\circ} \mathrm{C}$ & Abcam & ab153805 \\
GGPS1 & Rabbit & $1: 1000$ & $16 \mathrm{~h}, 4^{\circ} \mathrm{C}$ & Abcam & ab167168 \\
RCE1 & Rabbit & $1: 500$ & $16 \mathrm{~h}, 4^{\circ} \mathrm{C}$ & Abcam & ab62531 \\
CMT & Rabbit & $1: 500$ & $16 \mathrm{~h}, 4^{\circ} \mathrm{C}$ & Abcam & ab80872 \\
VCP & Mouse & $1: 25,000$ & $1 \mathrm{~h}, \mathrm{RT}$ & Abcam & ab11433 \\
VCP & Rabbit & $1: 25,000$ & $1 \mathrm{~h}, \mathrm{RT}$ & Abcam & ab109240 \\
\hline
\end{tabular}

\section{Western blot analysis}

Thawed homogenates were denatured at $70^{\circ} \mathrm{C}$ for 10 min under reducing conditions and stored at $-20^{\circ} \mathrm{C}$. Duplicate samples were loaded onto NuPAGE 4-12\% BisTris gels (Invitrogen, Carlsbad, CA) and transferred to nitrocellulose membranes using a BioRad Semi-Dry Transblotter (Hercules, CA). Membranes were incubated in Odyssey blocking buffer (LI-COR, Lincoln, NE) for $1 \mathrm{~h}$ at room temperature (RT) before being probed with the primary antibody diluted in LI-COR blocking buffer with $0.1 \%$ Tween-20, using the conditions indicated in Table 2. After incubation in primary antibody, membranes were washed in cold Tris-buffered saline $+0.05 \%$ Tween-20 (TBST) before being probed with IR-dyelabeled secondary antibody diluted in LI-COR blocking buffer $+0.1 \%$ Tween- 20 for $1 \mathrm{~h}$ at RT. Finally, membranes were washed in cold TBST, then briefly rinsed in MilliQ water before being scanned with a LI-COR Odyssey imager. All antibodies were optimized for ideal conditions of each target protein within the linear range of detection for each assay and ensuring the primary antibody was present in excess (Table 2). Valosin-containing protein (VCP) has been shown to be unchanged in multiple regions of schizophrenia brain ${ }^{32,33}$ and was used as an intralane loading control for western blot normalization.

\section{Data analysis}

Protein expression was determined using the LI-COR Odyssey 3.0 analytical software (Lincoln, NE). Intensity values were normalized to the intralane $\mathrm{VCP}$ intensity 
value after verifying that VCP was not changed between these schizophrenia and comparison subjects, consistent with previous reports ${ }^{32}$. Duplicate values were averaged for each subject. All dependent measures were tested for normal distribution with the D'Agostino-Pearson omnibus normality test. Normally distributed data were analyzed using two-tailed paired Student's $t$ tests, and Wilcoxon matched-pairs signed rank tests were used for non-normally distributed data using the GraphPad Prism software (GraphPad Software, La Jolla, CA). No dependent measures were found to be associated with age, $\mathrm{pH}$, or postmortem interval using post hoc linear regression analyses. For all statistical tests, $\alpha=0.05$.

\section{Bioinformatic analysis}

We evaluated prenylation-associated targets for patterns of differential gene expression in schizophrenia using six publically available transcriptomic datasets generated from samples from the MSSM NIH Brain and Tissue Repository. These datasets include studies in gray matter homogenates from the middle temporal area (BA 21), temporopolar area (BA 38), anterior cingulate cortex (BA 32), and DLPFC (BA 46) ${ }^{34}$. Two datasets examined transcript levels in laser capture microdissected pyramidal neurons from superficial (lamina II-III) or deep (lamina $\mathrm{V}-\mathrm{VI})$ cortical layers of the DLPFC ${ }^{35}$. The data were processed and analyzed using various $\mathrm{R}$ packages for differential expression analysis such as edgeR, DESeq2, and limma ${ }^{36-38}$. The datasets are then aggregated and uniquely titled for processing. To visualize patterns of differential expression, a heatmap of $\log 2$ fold change values has been constructed to present harmonized data across all of the different datasets. Harmonization was achieved using empirical cumulative probabilities based on each dataset, and final harmonized values were presented as standardized values that range from -1 to $1^{39}$. The unsupervised clustering and construction of heatmaps were done using the pheatmap $\mathrm{R}$ package ${ }^{40}$.

\section{Results}

\section{Prenyltransferase subunits are abnormally expressed in} schizophrenia

We found that each prenyltransferase enzyme had decreased expression of either or both of its respective $\alpha$ and $\beta$ subunits in schizophrenia relative to comparison subjects. The FTase and GGTase I $\alpha$ subunit, FNTA, was decreased $14 \%(t(12)=3.74, p=0.003)$. The GGTase I $\beta$ subunit PGGT1B was decreased 13\% $(W=-77, p=$ 0.004 ) and the Rab protein-specific GGTase II $\beta$ subunit RABGGTB was decreased $8 \%(t(12)=2.29, p=0.04)$ in schizophrenia (Fig. 1). We also assayed FNTA, PGGT1B, and RABGGTB in rats chronically treated with haloperidol decanoate and found that haloperidol treatment did not affect the expression of these proteins in these rats (Fig. 2).
Expression of upstream isoprenoid synthases and downstream prenylation-dependent enzymes are normal in schizophrenia

To determine whether upstream lipid donor synthesis of FPP or GGPP was altered in schizophrenia, we assayed the synthases for these lipid pyrophosphate molecules, FDPS and GPPS1. No differences in the expression of FDPS or GPPS1 were identified in schizophrenia (Table 3). To determine whether enzymes downstream of prenyl attachment that are involved in the secondary processing of prenylproteins were altered, we measured the protein expression of RCE and ICMT in schizophrenia and found normal expression of both (Table 3).

Transcript levels of prenylation-associated enzymes and prenylated substrates are altered in schizophrenia

Bioinformatic analysis of transcriptomic datasets generated from samples from the MSSM NIH Brain and Tissue Repository revealed that genes associated with prenylation demonstrate altered patterns of gene expression in schizophrenia. Genes encoding for upstream prenyl synthases, prenyltransferase subunits, prenylcysteine-processing enzymes, and some GTPases (substrates of prenylation) exhibit differential expression relative to comparison subjects in one or more of the datasets evaluated (Supplementary Fig. S1, Supplementary Table S1).

\section{Discussion}

Neurotransmission, synaptic plasticity, dendritic dynamics, and protein subcellular localization have all been reported to be abnormal in schizophrenia. Prenylation is a cytosolic PTM that enables many GTPases associated with these processes to correctly localize for signaling transduction ${ }^{16,19,21,26,41-44}$. GTPases have been shown to require combinations of lipid modifications including S-palmitoylation, N-myristoylation, and prenylation, which facilitate membrane-dependent GTPase activity $^{20-24}$. Given that a deficit in protein Spalmitoylation has been reported in schizophrenia ${ }^{45}$, we hypothesized that abnormal prenylation may also contribute to altered G-protein signaling pathways implicated in the illness ${ }^{6,17-19}$. We found protein expression of FNTA, PGGT1B, and RABGGTB prenyltransferase subunits decreased in schizophrenia DLPFC relative to paired comparison subjects, changes not likely due to chronic antipsychotic treatment. Bioinformatic assessments identified patterns of differential gene expression of prenylation-associated enzymes and substrates in schizophrenia. For individual genes, the direction and magnitude of differences appears to vary by brain region and cortical layer; however, identification of prenylationassociated differences across multiple datasets suggests that this functional pathway is involved in this illness. Together, these data are consistent with our previous 


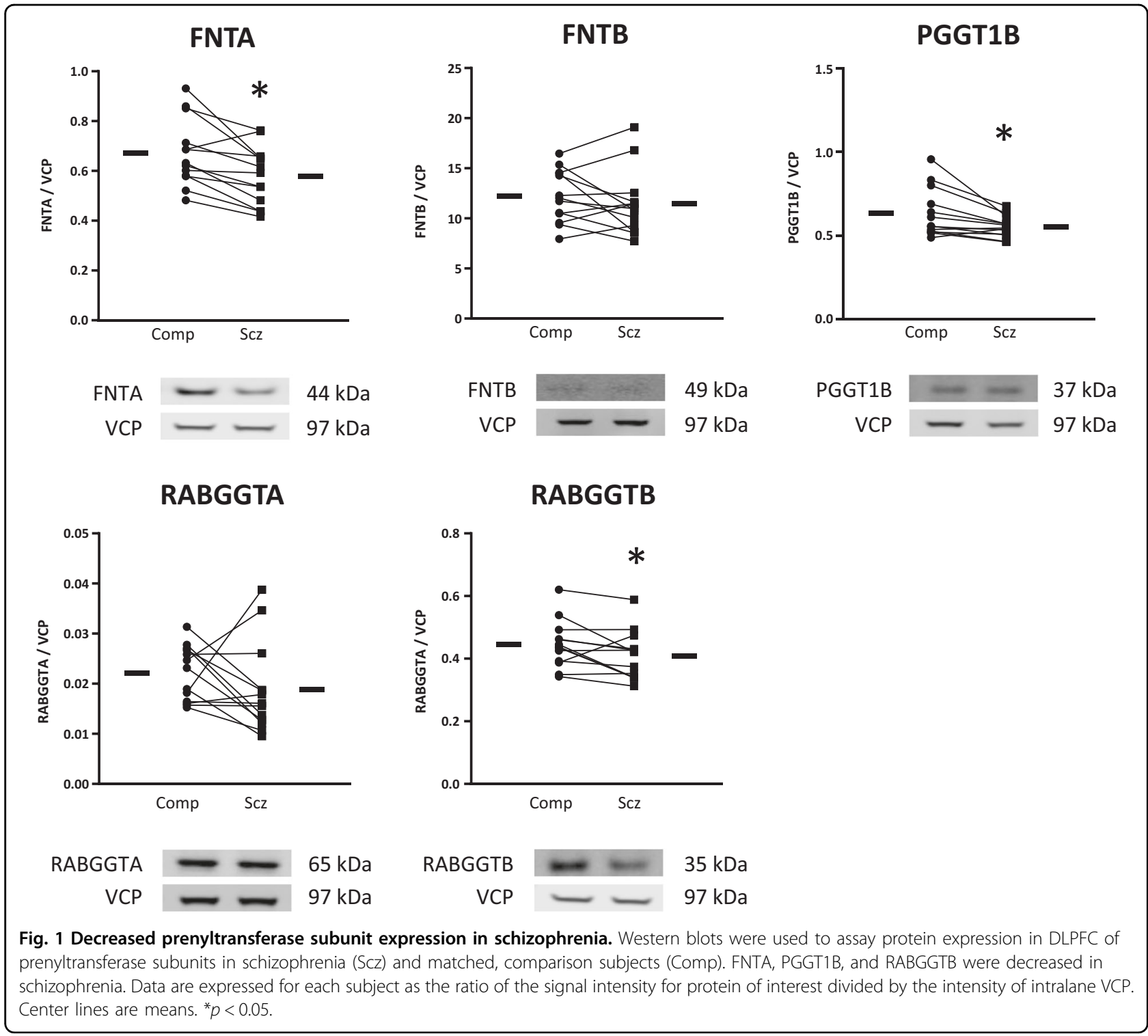

findings of abnormal lipid modifications in schizophrenia, including abnormal S-palmitoylation and decreased expression of an $\mathrm{N}$-myristoylated protein in schizophrenia DLPFC $^{11,12}$.

Given that FNTA, PGGT1B, and RABGGTB were decreased, each prenyltransferase enzyme $\alpha \beta$ complex then has at least one abnormally expressed subunit, and GGTase I has reduced expression of both its $\alpha$ and $\beta$ subunits. Previous reports demonstrated transcript-level upregulation of two of these subunits, FNTA and RABGGTB, in schizophrenia superior temporal gyrus (BA $22)^{46}$ and prefrontal cortex (BAs 9 and 10) ${ }^{47}$, respectively. These changes reported for transcript expression are in the opposite direction of the protein expression changes identified in the current study, which might suggest that upstream or downstream regulatory molecules may also be altered in schizophrenia, or may reflect cellular compensation.

Since upstream or downstream factors could be driving the changes in prenyltransferase expression, we also assayed protein expression of the isoprenoid synthases, FDPS and GGPS1, and prenylprotein-processing enzymes, RCE and ICMT. FDPS and GGPS1 catalyze the production of the key intermediates in the mevalonate pathway that are the lipid donors for farnesylation and geranylgeranylation, FPP and GGPP, which are attached to proteins by their respective prenyltransferase $(s)^{26}$. Following cytosolic prenylation, many prenylproteins are targeted to the endoplasmic reticulum, where they are subject to additional processing steps before they can ultimately be trafficked to the correct membrane destination $^{25}$. For substrates of FTase and GGTase I that 


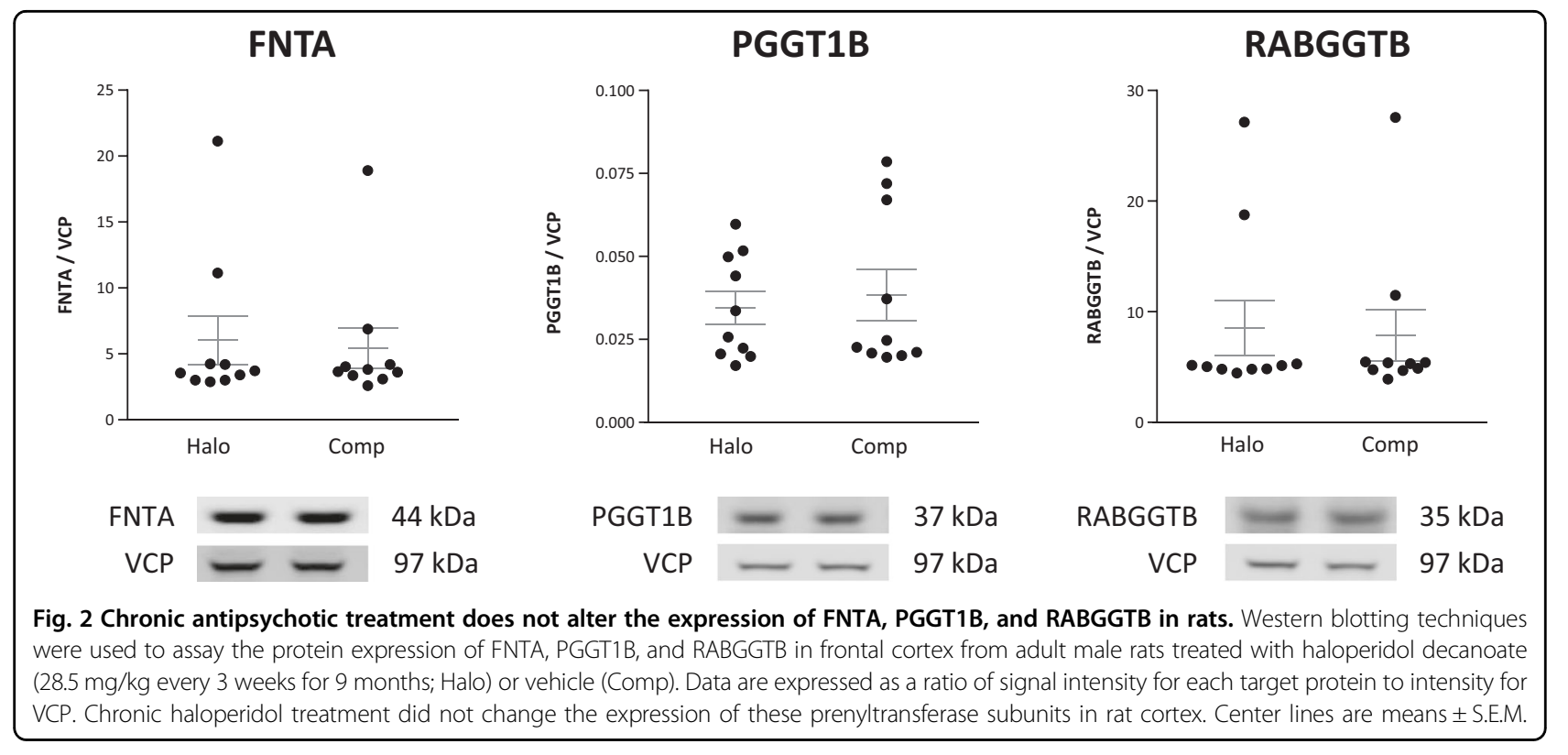

Table 3 Prenylation-associated enzyme expression levels in schizophrenia and comparison subjects.

\begin{tabular}{lccll}
\hline & Comparison & Schizophrenia & Test statistic & $\boldsymbol{p}$ value \\
\hline Prenylsynthases & & & \\
FDPS & $0.649 \pm 0.067$ & $0.641 \pm 0.067$ & $t(12)=0.35$ & 0.73 \\
GGPS1 & $0.194 \pm 0.066$ & $0.189 \pm 0.059$ & $t(12)=0.36$ & 0.72 \\
Prenyltransferase subunits & & & \\
FNTA & $0.673 \pm 0.136$ & $0.580 \pm 0.117$ & $t(12)=3.74$ & $0.003^{*}$ \\
FNTB & $12.24 \pm 2.618$ & $11.47 \pm 3.235$ & $W=-25$ & 0.41 \\
PGGT1B & $0.633 \pm 0.146$ & $0.553 \pm 0.063$ & $W=-77$ & $0.004^{*}$ \\
RABGGTA & $0.022 \pm 0.006$ & $0.019 \pm 0.009$ & $t(12)=1.14$ & 0.28 \\
RABGGTB & $0.445 \pm 0.076$ & $0.409 \pm 0.078$ & $t(12)=2.29$ & $0.04^{*}$ \\
Prenylcysteine-processing enzymes & & \\
RCE & $0.127 \pm 0.038$ & $0.119 \pm 0.033$ & $W=-11$ & 0.74 \\
ICMT & $0.197 \pm 0.061$ & $0.188 \pm 0.055$ & $t(12)=0.68$ & 0.51 \\
\hline
\end{tabular}

Comparison and schizophrenia values are reported as means \pm S.D. ${ }^{*} p \leq 0.05$

contain a C-terminal "CAAX" amino acid sequence (where $\mathrm{C}$ is cysteine, $\mathrm{A}$ is any aliphatic amino acid, and $\mathrm{X}$ is any amino acid), RCE cleaves the AAX residues from the prenyl-cysteine, and ICMT subsequently catalyzes the methylation of the prenyl-cysteine ${ }^{25}$. These upstream and downstream enzymes, however, were not found to be differently expressed in schizophrenia DLPFC. Together, these data suggest that isoprenoid lipid donor synthesis and prenyl-cysteine modifications that occur following prenylation are normal in schizophrenia, but the actual attachment of prenyl groups to protein substrates could be impaired.

Many prenylproteins belong to the superfamily of Gproteins, which play key roles in synaptic regulation. Heterotrimeric G-protein $\alpha, \beta$, and $\gamma$ subunits require lipid modifications for membrane targeting, protein interaction, and activity. $G \alpha$ subunits can be $\mathrm{N}$ myristoylated and/or S-palmitoylated ${ }^{14,22,48}$, and Gy subunits are prenylated ${ }^{16}$. Prenylation is also required for some proteins involved with the regulation of $\mathrm{Ca}^{2+}$ signaling, spinogenesis, and synaptogenesis ${ }^{49,50}$, pathways which have been implicated in schizophrenia ${ }^{18,43,51,52}$.

Many members of the Ras protein superfamily of small GTPases, which include Ras, Rho, Rab, Rap, Arf, Ran, and Rheb subfamilies, are also prenylated ${ }^{4}$. The Rab and Rho protein subfamilies regulate membrane trafficking and cytoskeletal dynamics, as well as spatiotemporal aspects of vesicular recycling within the $\mathrm{cell}^{53}$. These proteins require prenylation for correct interactions with their effector proteins at cell membranes ${ }^{54,55}$. Ras subfamily GTPases are involved in signal transduction and the regulation of gene expression ${ }^{56}$ and recent studies suggest that Ras family protein signaling plays a role in memory formation ${ }^{57}$. Given that pharmacological inhibition of Ras farnesylation prevents signal transduction to downstream targets in cell culture ${ }^{58}$, decreased expression of functional FTases could inhibit the interaction of Ras proteins with their effectors and contribute to altered intracellular signaling and working memory in schizophrenia. Agerelated downregulation of PGGT1B in mice is associated with decreased membrane-associated Rho-GTPases involved in synaptic plasticity. Inhibition of GGTase I in vitro leads to decreased protein levels of synaptophysin 
and GAP- $43^{59}$, and these proteins are also decreased in schizophrenia brain ${ }^{60-62}$. Studies using statins, which inhibit the activity of the mevalonate pathway that produces FPP and GGPP, and prenylation-specific inhibitors in neuronal cultures, have reported decreased dendritic aborization $^{63,64}$ and increased axonal growth ${ }^{65,66}$. These reports emphasize that morphological abnormalities can arise from altered prenylation and suggest one potential mechanism contributing to dendritic spine abnormalities in schizophrenia brain ${ }^{43,67}$.

Often the isoprenyl PTM by itself is not sufficient to maintain a strong membrane association, and many prenylated proteins require a second signal for membrane localization. This second signal is typically the addition of a palmitoyl group or the presence of a polybasic domain $^{4,68}$. We have previously reported a deficit of protein S-palmitoylation in schizophrenia ${ }^{11}$, and it is likely that the subset of proteins which require both prenylation and S-palmitoylation are more susceptible to dysfunction if both of these pathways are impaired. Some members of the Ras subfamily are known to be both prenylated and S-palmitoylated, with prenylation occurring upstream of S-palmitoylation ${ }^{4,27,28}$. Our prior report of decreased S-palmitoylation identified a widespread decrease of S-palmitoylation across many proteins, including Ras, but did not identify alterations in the expression levels of enzymes that attach or cleave palmitoyl groups that would explain the reduction ${ }^{11}$. Our current finding of reduced prenyltransferase expression suggests that defective prenylation of Ras could potentially prevent the appropriate S-palmitoylation of these molecules, consistent with the findings of our previous study. If this is indeed the case, altered Ras lipidation might impair correct intracellular localization and/or signaling of these small GTPases. Deficits in DLPFC function, circuitry, and working memory have been repeatedly implicated in schizophrenia ${ }^{69,70}$. Considering that Ras family signaling contributes to memory formation $^{57}$, abnormal lipid modifications in this brain region may contribute to memory-associated deficits in the disorder.

Postmortem brain studies in schizophrenia inherently have limitations. We evaluated gene expression differences and measured protein expression levels in aged subjects, and these data may not generalize to different age groups or earlier stages of the illness. Our protein study is also restricted to the DLPFC, thus additional brain regions will need to be examined to determine whether these findings are widespread or brain region specific. Furthermore, given that some enzymes measured in this study demonstrate cell-type-specific patterns of expression, prenylation pathway alterations identified here may preferentially impact a distinct subpopulation of cells and cell-type-specific protein measures will be necessary to investigate this possibility. Another major limitation of schizophrenia postmortem studies is that chronic antipsychotic treatment may affect the expression of some proteins independent of the disorder. To rule out the potential effects of long-term antipsychotic use, we assayed these proteins in frontal cortex of rats chronically treated with haloperidol decanoate. We did not find any effects of long-term haloperidol treatment on protein levels of FNTA, PGGT1B, or RABGGTB, which suggests that reduced prenyltransferase subunit expression identified in schizophrenia is likely due to the illness and not a result of chronic antipsychotic treatment. However, it is important to note that the haloperidol studies were not performed in an animal model of schizophrenia, and potential interactions between disease pathophysiology and long-term antipsychotic use that may influence protein prenylation cannot be completely ruled out.

In summary, we found decreased protein expression of the prenyltransferase subunits FNTA, PGGT1B, and RABGGTB in schizophrenia DLPFC as well as evidence from a bioinformatic analysis of differential gene expression of prenylation-associated genes across multiple brain regions and cortical layers. These data are consistent with other evidence of abnormal lipid modifications in schizophrenia $^{11,12}$ and suggest a potential mechanism for our report of reduced Ras S-palmitoylation in the face of normal palmitoyltransferase expression. Because of its importance in G-protein signaling and small GTPase activity, abnormal prenylation is also a potential mechanism underlying altered intracellular signaling, dendritic dynamics, and subcellular protein localization previously reported in schizophrenia. Decreased protein expression of these prenyltransferase subunits could contribute to many facets of the pathophysiology of schizophrenia by its important role in multiple cell biological processes.

\section{Acknowledgements \\ The authors gratefully acknowledge Dr. Rosalinda Roberts and the Alabama Brain Collection for providing materials used to optimize conditions used in this study.}

Conflict of interest

The authors declare that they have no conflict of interest.

\section{Publisher's note}

Springer Nature remains neutral with regard to jurisdictional claims in published maps and institutional affiliations.

Supplementary Information accompanies this paper at (https://doi.org/ 10.1038/s41398-019-0610-7).

Received: 31 October 2018 Revised: 9 September 2019 Accepted: 3 October 2019

Published online: 10 January 2020 


\section{References}

1. Hamm, H. E. The many faces of $G$ protein signaling. J. Biol. Chem. 273, 669-672 (1998).

2. Ferguson, S. S. Evolving concepts in G protein-coupled receptor endocytosis: the role in receptor desensitization and signaling. Pharmacol. Rev. 53, 1-24 (2001).

3. Resh, M. D. Trafficking and signaling by fatty-acylated and prenylated proteins. Nat. Chem. Biol. 2, 584-590 (2006).

4. Resh, M. D. Covalent lipid modifications of proteins. Curr. Biol. 23, R431-R435 (2013).

5. Resh, M. D. Palmitoylation of ligands, receptors, and intracellular signaling molecules. Sci. STKE 2006, re14 (2006).

6. Mirnics, K., Middleton, F. A., Stanwood, G. D., Lewis, D. A. \& Levitt, P. Diseasespecific changes in regulator of G-protein signaling 4 (RGS4) expression in schizophrenia. Mol. Psychiatry 6, 293-301 (2001).

7. Hottman, D. A. \& Li, L. Protein prenylation and synaptic plasticity: implications for Alzheimer's disease. Mol. Neurobiol. 50, 177-185 (2014).

8. Yanai, A. et al. Palmitoylation of huntingtin by HIP14 is essential for its trafficking and function. Nat. Neurosci. 9, 824-831 (2006).

9. Singaraja, R. R. et al. Altered palmitoylation and neuropathological deficits in mice lacking HIP14. Hum. Mol. Genet. 20, 3899-3909 (2011).

10. Mukai, J. et al. Palmitoylation-dependent neurodevelopmental deficits in a mouse model of 22q11 microdeletion. Nat. Neurosci. 11, 1302-1310 (2008).

11. Pinner, A. L., Tucholski, J., Haroutunian, V., McCullumsmith, R. E. \& MeadorWoodruff, J. H. Decreased protein S-palmitoylation in dorsolateral prefrontal cortex in schizophrenia. Schizophr. Res. 177, 78-87 (2016).

12. Pinner, A. L., Haroutunian, V. \& Meador-Woodruff, J. H. Alterations of the myristoylated, alanine-rich C kinase substrate (MARCKS) in prefrontal cortex in schizophrenia. Schizophr. Res. 154, 36-41 (2014).

13. Buss, J. E., Mumby, S. M., Casey, P. J., Gilman, A. G. \& Sefton, B. M. Myristoylated alpha subunits of guanine nucleotide-binding regulatory proteins. Proc. Natl Acad. Sci. USA 84, 7493-7497 (1987).

14. Chen, C. A. \& Manning, D. R. Regulation of $G$ proteins by covalent modification. Oncogene 20, 1643-1652 (2001).

15. Escriba, P. V., Wedegaertner, P. B., Goni F. M. \& Vogler O. Lipid-protein interactions in GPCR-associated signaling. Biochim. Biophys. Acta 1768, 836-852 (2007).

16. Higgins, J. B. \& Casey, P. J. The role of prenylation in G-protein assembly and function. Cell. Signal. 8, 433-437 (1996).

17. Bychkov, E. R., Ahmed, M. R., Gurevich, V. V., Benovic, J. L. \& Gurevich, E. V. Reduced expression of $\mathrm{G}$ protein-coupled receptor kinases in schizophrenia but not in schizoaffective disorder. Neurobiol. Dis. 44, 248-258 (2011).

18. Catapano L. A., Manji H. K. G protein-coupled receptors in major psychiatric disorders. Biochim. Biophys. Acta 1768, 976-993 (2007).

19. Datta, D., Arion, D., Corradi, J. P. \& Lewis, D. A. Altered expression of CDC42 signaling pathway components in cortical layer 3 pyramidal cells in schizophrenia. Biol. Psychiatry 78, 775-785 (2015).

20. Casey, P. J. Lipid modifications of G proteins. Curr. Opin. cell Biol. 6, 219-225 (1994).

21. Casey, P. J., Moomaw, J. F., Zhang, F. L., Higgins, Y. B. \& Thissen, J. A. Prenylation and G protein signaling. Recent Prog. Horm. Res. 49, 215-238 (1994).

22. Wedegaertner, P. B., Wilson, P. T. \& Bourne, H. R. Lipid modifications of trimeric G proteins. J. Biol. Chem. 270, 503-506 (1995).

23. Milligan, G., Parenti, M. \& Magee, A. I. The dynamic role of palmitoylation in signal transduction. Trends Biochem. Sci. 20, 181-187. (1995).

24. McCallum, J. F. et al. The role of palmitoylation of the guanine nucleotide binding protein $\mathrm{G} 11$ alpha in defining interaction with the plasma membrane. Biochem. J. 310(Pt 3), 1021-1027 (1995).

25. Clarke, S. Protein isoprenylation and methylation at carboxyl-terminal cysteine residues. Annu. Rev. Biochem. 61, 355-386 (1992).

26. Zhang, F. L. \& Casey, P. J. Protein prenylation: molecular mechanisms and functional consequences. Annu. Rev. Biochem. 65, 241-269 (1996).

27. Rocks, $\mathrm{O}$. et al. The palmitoylation machinery is a spatially organizing system for peripheral membrane proteins. Cell 141, 458-471 (2010).

28. Resh, M. D. Targeting protein lipidation in disease. Trends Mol. Med. 18, 206-214 (2012)

29. Powchik, P. et al. Postmortem studies in schizophrenia. Schizophr. Bull. 24, 325-341 (1998)

30. Harte, M. K., Bachus, S. B. \& Reynolds, G. P. Increased N-acetylaspartate in rat striatum following long-term administration of haloperidol. Schizophr. Res. 75 303-308 (2005)
31. Kashihara, K. et al. Effects of intermittent and continuous haloperidol administration on the dopaminergic system in the rat brain. Biol. Psychiatry 21 650-656 (1986).

32. Bauer, D. E., Haroutunian, V., McCullumsmith, R. E. \& Meador-Woodruff, J. H. Expression of four housekeeping proteins in elderly patients with schizophrenia. J. Neural Transm. 116, 487-491 (2009).

33. Stan, A. D. et al. Human postmortem tissue: what quality markers matter? Brain Res. 1123, 1-11 (2006).

34. Roussos, P., Katsel, P., Davis, K. L., Siever, L. J. \& Haroutunian, V. A system-level transcriptomic analysis of schizophrenia using postmortem brain tissue samples. Arch. Gen. Psychiatry 69, 1205-1213 (2012).

35. Sullivan, C. R. et al. Neuron-specific deficits of bioenergetic processes in the dorsolateral prefrontal cortex in schizophrenia. Mol. Psychiatry 24, 1319-1328 (2018).

36. Robinson, M. D., McCarthy, D. J. \& Smyth, G. K. edgeR: a Bioconductor package for differential expression analysis of digital gene expression data. Bioinformatics 26, 139-140 (2010).

37. Love, M. I., Huber, W. \& Anders, S. Moderated estimation of fold change and dispersion for RNA-seq data with DESeq2. Genome Biol. 15, 550 (2014).

38. Ritchie, M. E. et al. limma powers differential expression analyses for RNAsequencing and microarray studies. Nucleic Acids Res. 43, e47 (2015).

39. Rouillard, A. D. et al. The harmonizome: a collection of processed datasets gathered to serve and mine knowledge about genes and proteins. Database (Oxford) 2016, baw100 (2016).

40. Kolde, R. pheatmap: pretty heatmaps. R package version 1.0.10, https://www. rdocumentation.org/packages/pheatmap/versions/1.0.10 (2018).

41. Moghaddam, B. \& Javitt, D. From revolution to evolution: the glutamate hypothesis of schizophrenia and its implication for treatment. Neuropsychopharmacology 37, 4-15 (2012).

42. Mueller, T. M., Remedies, C. E., Haroutunian, V. \& Meador-Woodruff, J. H. Abnormal subcellular localization of GABAA receptor subunits in schizophrenia brain. Transl. Psychiatry 5, e612 (2015).

43. Ide, M. \& Lewis, D. A. Altered cortical CDC42 signaling pathways in schizophrenia: implications for dendritic spine deficits. Biol. Psychiatry 68, 25-32 (2010).

44. Stephan, K. E., Baldeweg, T. \& Friston, K. J. Synaptic plasticity and dysconnection in schizophrenia. Biol. Psychiatry 59, 929-939 (2006).

45. Pinner, A. L., Tucholski, J., Haroutunian, V., McCullumsmith, R. E. \& MeadorWoodruff, J. H. Decreased protein S-palmitoylation in dorsolateral prefrontal cortex in schizophrenia. Schizophr. Res. 177, 78-87 (2016).

46. Bowden, N. A., Scott, R. J. \& Tooney, P. A. Altered gene expression in the superior temporal gyrus in schizophrenia. BMC Genomics 9, 199 (2008).

47. Maycox, P. R. et al. Analysis of gene expression in two large schizophrenia cohorts identifies multiple changes associated with nerve terminal function. Mol. Psychiatry 14, 1083-1094 (2009).

48. Wedegaertner, P. B. Lipid modifications and membrane targeting of $\mathrm{G}$ alpha. Biol. Signals Recept. 7, 125-135 (1998).

49. Tolias, K. F., Duman, J. G. \& Um, K. Control of synapse development and plasticity by Rho GTPase regulatory proteins. Prog. Neurobiol. 94, 133-148 (2011).

50. Cullen, P. J. \& Lockyer, P. J. Integration of calcium and Ras signalling. Nat. Rev Mol. Cell Biol. 3, 339-348 (2002).

51. Lidow, M. S. Calcium signaling dysfunction in schizophrenia: a unifying approach. Brain Res. Brain Res. Rev. 43, 70-84 (2003).

52. Datta, D., Arion, D., Corradi, J. P. \& Lewis, D. A. Altered expression of CDC42 signaling pathway components in cortical layer 3 pyramidal cells in schizophrenia. Biol. Psychiatry 78, 775-785 (2015).

53. Takai, Y., Sasaki, T., Tanaka, K. \& Nakanishi, H. Rho as a regulator of the cytoskeleton. Trends Biochem. Sci. 20, 227-231 (1995).

54. Seabra, M. C. Membrane association and targeting of prenylated Ras-like GTPases. Cell Signal. 10, 167-172 (1998).

55. Nishimura, A. \& Linder, M. E. Identification of a novel prenyl and palmitoyl modification at the CaaX motif of Cdc42 that regulates RhoGDI binding. Mol. Cell. Biol. 33, 1417-1429 (2013).

56. Hancock, J. F. Ras proteins: different signals from different locations. Nat. Rev. Mol. Cell Biol. 4, 373-384. (2003).

57. Ye, X. \& Carew, T. J. Small G protein signaling in neuronal plasticity and memory formation: the specific role of ras family proteins. Neuron $\mathbf{6 8}, 340-361$ (2010). 
58. Cox, A. D. et al. The CAAX peptidomimetic compound B581 specifically blocks farnesylated, but not geranylgeranylated or myristylated, oncogenic ras signaling and transformation. J. Biol. Chem. 269, 19203-19206 (1994).

59. Afshordel, S., Wood, W. G., Igbavboa, U., Muller, W. E. \& Eckert, G. P. Impaired geranylgeranyltransferase-I regulation reduces membraneassociated Rho protein levels in aged mouse brain. J. Neurochem. 129, 732-742 (2014).

60. Tian, S. Y., Wang, J. F., Bezchlibnyk, Y. B. \& Young, L. T. Immunoreactivity of $43 \mathrm{kDa}$ growth-associated protein is decreased in post mortem hippocampus of bipolar disorder and schizophrenia. Neurosci. Lett. 411, 123-127 (2007).

61. Fung, S. J., Sivagnanasundaram, S. \& Weickert, C. S. Lack of change in markers of presynaptic terminal abundance alongside subtle reductions in markers of presynaptic terminal plasticity in prefrontal cortex of schizophrenia patients. Biol. Psychiatry 69, 71-79 (2011).

62. Glantz, L. A. \& Lewis, D. A. Reduction of synaptophysin immunoreactivity in the prefrontal cortex of subjects with schizophrenia. Regional and diagnostic specificity. Arch. Gen. Psychiatry 54, 943-952 (1997)
63. Kim, W. Y. et al. Statins decrease dendritic arborization in rat sympathetic neurons by blocking RhoA activation. J. Neurochem. 108, 1057-1071 (2009).

64. Schulz, J. G. et al. HMG-CoA reductase inhibition causes neurite loss by interfering with geranylgeranylpyrophosphate synthesis. J. Neurochem. 89, 24-32 (2004).

65. $\mathrm{Li}, \mathrm{H}$. et al. Protein prenylation constitutes an endogenous brake on axonal growth. Cell Rep. 16, 545-558 (2016).

66. Pooler, A. M., Xi, S. C. \& Wurtman, R. J. The 3-hydroxy-3-methylglutaryl coenzyme $A$ reductase inhibitor pravastatin enhances neurite outgrowth in hippocampal neurons. J. Neurochem. 97, 716-723 (2006).

67. Glausier, J. R. \& Lewis, D. A. Dendritic spine pathology in schizophrenia. Neuroscience 251, 90-107 (2013).

68. Hancock, J. F., Paterson, H. \& Marshall, C. J. A polybasic domain or palmitoylation is required in addition to the CAAX motif to localize p21ras to the plasma membrane. Cell 63, 133-139 (1990).

69. Lewis, D. A. \& Gonzalez-Burgos, G. Neuroplasticity of neocortical circuits in schizophrenia. Neuropsychopharmacology 33, 141-165 (2008).

70. Potkin, S. G. et al. Working memory and DLPFC inefficiency in schizophrenia: the FBIRN study. Schizophr. Bull. 35, 19-31 (2009). 